\title{
立方相 $\mathrm{Y}$ 掺杂 $\mathrm{ZrO}_{2}$ 纳米晶薄膜的制备与辐照效应研究
}

\author{
王卡, 万康, 张静, 陈林, 陈琼, 常永勤, 龙毅 \\ (北京科技大学 材料科学与工程学院, 北京 100083)
}

摘 要: 采用溶胶-凝胶结合旋涂法在单晶 $\mathrm{Si}$ 祄底上制备了立方相 $\mathrm{Y}$ 掺杂 $\mathrm{ZrO}_{2}$ 纳米晶薄膜( $\mathrm{YSZ}$, 并分析了制备工 艺参数对 YSZ 成膜的影响。采用光学显微镜、扫描电子显微镜、X 射线衍射和透射电镜等手段对样品进行了表征 和分析。结果表明, 加入 PVA 作为分散剂、采用分级干燥工艺以及提高匀胶转速可大大提高 YSZ 薄膜的成膜质量, 制备的 YSZ 薄膜表面十分平整, 没有出现裂纹。YSZ 薄膜为立方相结构, 没有出现其它相。薄膜由平均晶粒尺寸 为 $9.4 \mathrm{~nm}$ 的纳米晶组成, 薄膜的厚度约为 $60 \mathrm{~nm}$ 。在室温条件下, 低剂量的 Xe 离子辐照 $\mathrm{YSZ}$ 薄膜后出现微裂纹, 而 当辐照剂量比较高时, 由于热峰效应, 辐照引起的微裂纹逐渐发生愈合。并且, 随着辐照剂量的增加, YSZ 薄膜的 平均晶粒尺寸增大。

关 键 词: $\mathrm{ZrO}_{2}$ 纳米晶薄膜; 溶胶-凝胶法; 立方相; 辐照效应

中图分类号: TB43 文献标识码: A

\section{Fabrication and Irradiation of Nanocrystalline Yttrium-stabilized Cubic $\mathrm{ZrO}_{2}$ Film}

\author{
WANG Ka, WAN Kang, ZHANG Jing, CHEN Lin, CHEN Qiong, CHANG Yong-Qin, LONG Yi
}

(School of Materials Science and Engineering, University of Science and Technology Beijing, Beijing 100083, China)

\begin{abstract}
Nanocrystalline cubic yttrium-stabilized $\mathrm{ZrO}_{2}$ films (YSZ) were prepared on silicon substrate via Sol-Gel combined with spin-coating method. The as-grown films were characterized by optical microscope, SEM, XRD and TEM. It was found that preparation parameters have obvious effect on quality of the YSZ film. The quality of YSZ thin films can be greatly improved by adding PVA as dispersing agent, adopting the grading drying process and increasing the spin speed. Smooth films without any cracks can be obtained by optimum growth conditions. XRD result shows that the film is cubic structure with no other phase. The thickness of the YSZ film is about $60 \mathrm{~nm}$, and its average grain-size is $9.4 \mathrm{~nm}$. When the samples are irradiated by Xe ions with low dose at room-temperature, microscopic cracks are detected in the YSZ films. As the ion irradiation dose increases, the irradiation-induced cracks are gradually recovered, which might attribute to the thermal spike effect on the film. In addition, the average grain-size of the YSZ film grows with irradiation dose increasing.
\end{abstract}

Key words: nanocrystalline $\mathrm{ZrO}_{2}$ film; Sol-Gel; cubic phase; irradiation effect

用于核反应堆的二氧化铀、二氧化针、二氧化 钚等材料具有放射性, 研究这些材料在辐照环境 中的变化对研究条件要求非常苛刻, 同时对使用
环境也要求严格。立方相氧化锆 $\left(\mathrm{ZrO}_{2}\right)$ 与上述材料 同构，既没有放射性，还具有非常优良的抗辐照性 能，使其成为研究核反应堆在极端辐照环境中陶

收稿日期：2014-12-16; 收到修改稿日期：2015-02-05

基金项目 : 国家自然科学基金(50502005, 11175014); 新世纪优秀人才计划(NCET-07-0065) National Natural Science Foundation of China (50502005, 11175014); Program for New Century Excellent Talents in University (NCET-07-0065)

作者简介: 王 卡(1990-)，男，硕士研究生. E-mail: wangka200888@163.com

通讯作者: 常永勤, 教授. E-mail: chang@ustb.edu.cn 
瓷氧化物辐照行为的理想材料 ${ }^{[1-2]}$ 。晶界可以提供 吸收辐照引入缺陷的吸收源, 尤其是在晶面和晶 界处移动的点缺陷。纳米晶材料具有很高的晶界密 度, 使其可能具有良好的抗辐照性能而引起广泛 关注 ${ }^{[3-4]}$ 。室温下 $\mathrm{ZrO}_{2}$ 具有单斜晶体结构, 随着温 度的升高, 它会转变为四方相 $\left(\sim 1170^{\circ} \mathrm{C}\right)$ 和立方相 $\left(\sim 2370^{\circ} \mathrm{C}\right)$ 。 $\mathrm{ZrO}_{2}$ 的抗辐照性能与其晶型有密切关 系, 已有的研究结果表明 ${ }^{[5-7]}$, 立方相的纳米 $\mathrm{ZrO}_{2}$ 辐照后不发生相变, 辐照产生大量的氧空位使其 仍保持立方相, 同时伴随着晶粒的长大。而单斜相 的 $\mathrm{ZrO}_{2}$ 纳米晶辐照后会向四方相转变 ${ }^{[8-11]}$, 在高 能量的重离子辐照下甚至会发生非晶化 ${ }^{[12]}$ 。 Meldrum 等 ${ }^{[13-14]}$ 研究表明, 四方相纳米晶 $\mathrm{ZrO}_{2}$ 辐 照后会向非晶化转变。Lian 等 ${ }^{[15]}$ 对立方相和非晶 组成的双层 $\mathrm{ZrO}_{2}$ 薄膜进行离子辐照, 发现非晶向 四方相转变, 且立方相的晶粒长大速率明显小于 四方相。这些结果表明立方相的纳米 $\mathrm{ZrO}_{2}$ 具备优 良的抗辐照性能。目前对立方相纳米 $\mathrm{ZrO}_{2}$ 的辐照 研究一般为非掺杂立方相纳米 $\mathrm{ZrO}_{2}$, 对 $\mathrm{Y}$ 掺杂立 方相纳米晶 $\mathrm{ZrO}_{2}$ 的研究才刚刚起步。

立方相 $\mathrm{ZrO}_{2}$ 属于高温稳定相, 在室温条件下 一般不易获得。在 $\mathrm{ZrO}_{2}$ 晶体中掺入一定量的低价 阳离子 (如 $\mathrm{Y}^{3+} 、 \mathrm{Ca}^{2+}$ ) 替代 $\mathrm{Zr}^{4+}$, 为保持电中性而引 入了大量的氧空位, 从而在室温条件下获得稳定 的立方相 $\mathrm{ZrO}_{2}{ }^{[16]}$ 。另外, 通过制备纳米晶 $\mathrm{ZrO}_{2}$ 来 获得立方相的 $\mathrm{ZrO}_{2}$, 如一般采用离子束辅助沉积 (IBAD)方法来制备平均晶粒尺寸只有几个纳米的 立方相 $\mathrm{ZrO}_{2}$ 纳米晶薄膜 ${ }^{[3,5-7]}$, 这种方法获得的纳 米晶的晶粒大小比较均匀, 厚度容易控制, 但是其 操作工艺复杂, 成本较高, 而且可控性也比较 差。也有报道通过对 $\mathrm{ZrSiO}_{4}$ 进行离子束非晶化来 获得埋覆在非晶 $\mathrm{SiO}_{2}$ 中的 $\mathrm{ZrO}_{2}$ 纳米晶, 但是这 种方法制备出的 $\mathrm{ZrO}_{2}$ 纳米晶在辐照条件下容易 非晶化 ${ }^{[13-14]}$ 。溶胶一凝胶方法制备工艺比较简单, 成本低廉。采用溶胶一凝胶方法合成的 $\mathrm{ZrO}_{2}$ 纳米 晶大部分是粉体材料 ${ }^{[17-18]}$, 也有研究在祄底上 制备了 $\mathrm{ZrO}_{2}$ 薄膜 ${ }^{[19]}$, 但是仍然存在许多问题, 如在祄底上涂覆的薄膜容易发生开裂, 薄膜的平 整度差等。

本工作采用溶胶-凝胶结合旋涂的方法制备立 方相 $\mathrm{Y}$ 掺杂 $\mathrm{ZrO}_{2}$ 纳米晶薄膜, 探讨了工艺参数对 YSZ 成膜的影响, 并对薄膜的形貌和结构进行了 表征和分析, 研究了室温 Xe 离子辐照对 YSZ 纳米 晶薄膜的影响。

\section{1 实验方法}

\section{1 $\mathrm{ZrO}_{2}$ 薄膜的制备}

称取 $1.611 \mathrm{~g}$ 氧氯化锆 $\left(\mathrm{ZrOCl}_{2} \bullet 8 \mathrm{H}_{2} \mathrm{O}\right)$ 和 $0.332 \mathrm{~g}$ 硝酸钎 $\left(\mathrm{Y}\left(\mathrm{NO}_{3}\right)_{3} \cdot 6 \mathrm{H}_{2} \mathrm{O}\right)$ 溶于 $10 \mathrm{~mL}$ 去离子水中配成 $0.5 \mathrm{~mol} / \mathrm{L}$ 的溶液。称取 $0.70 \mathrm{~g}$ 草酸 $\left(\mathrm{H}_{2} \mathrm{C}_{2} \mathrm{O}_{4} \cdot 2 \mathrm{H}_{2} \mathrm{O}\right)$ 溶于 $15 \mathrm{~mL}$ 去离子水中配置成 $0.33 \mathrm{~mol} / \mathrm{L}$ 的溶液。 上述两种溶液均用磁力摚拌器搅拌使其充分溶解。 在室温下将氧氯化锆溶液缓慢滴加到草酸溶液中, 并持续搅拌, 得到淡蓝色透明溶胶, 然后向溶胶中 加入 $0.8 \mathrm{~mL}$ 的乙酰丙酮作为溶胶稳定剂。向溶胶中 加入 $5 \mathrm{wt} \%$ 的聚乙烯醇(PVA)溶液并摚拌均匀。将清 洗干净的硅片作为祄底, 用台式匀胶机进行涂膜, 转速为 $8000 \mathrm{r} / \mathrm{min}$, 涂膜时间为 $60 \mathrm{~s}$ 。涂膜后进行分级 干燥(先在 $80^{\circ} \mathrm{C}$ 干燥 $15 \mathrm{~min}$, 然后在 $50^{\circ} \mathrm{C}$ 下干燥 $30 \mathrm{~min}$, 最后在 $30^{\circ} \mathrm{C}$ 干燥 $30 \mathrm{~min}$ ), 之后在 $300^{\circ} \mathrm{C}$ 预烧结 $20 \mathrm{~min}$ 。 重复上述过程进行多次涂覆。将涂好膜的硅片放入 管式高温炉中 $500^{\circ} \mathrm{C}$ 退火 $1 \mathrm{~h}$ 后取出空冷。

在整个制备过程发生如下化学反应:

$$
\begin{aligned}
& \mathrm{ZrOCl}_{2}+\mathrm{H}_{2} \mathrm{C}_{2} \mathrm{O}_{4} \rightarrow \mathrm{ZrOC}_{2} \mathrm{O}_{4}+2 \mathrm{HCl} \\
& \mathrm{Y}\left(\mathrm{NO}_{3}\right)_{3}+\mathrm{H}_{2} \mathrm{C}_{2} \mathrm{O}_{4} \rightarrow \mathrm{Y}_{2}\left(\mathrm{C}_{2} \mathrm{O}_{4}\right)_{3}+2 \mathrm{HNO}_{3} \\
& \mathrm{ZrOC}_{2} \mathrm{O}_{4} \rightarrow \mathrm{ZrO}_{2}+2 \mathrm{CO}_{2} \uparrow \\
& \mathrm{Y}_{2}\left(\mathrm{C}_{2} \mathrm{O}_{4}\right)_{3} \rightarrow \mathrm{Y}_{2} \mathrm{O}_{3}+3 \mathrm{CO} \uparrow+3 \mathrm{CO}_{2} \uparrow \\
& (1-0.08) \mathrm{ZrO}_{2}+0.08 \mathrm{Y}_{2} \mathrm{O}_{3} \rightarrow\left(\mathrm{ZrO}_{2}\right)_{0.92}\left(\mathrm{Y}_{2} \mathrm{O}_{3}\right)_{0.08}
\end{aligned}
$$

其中, 乙酰丙酮的熔点约为 $-23^{\circ} \mathrm{C}$, 沸点约为 $140^{\circ} \mathrm{C}$, 在热处理过程中它能够完全挥发; PVA 的分解温度 也较低, 在空气中 PVA 开始分解温度约为 $230^{\circ} \mathrm{C}$, 当温度达到 $250^{\circ} \mathrm{C}$ 时几乎能完全分解，可见这两种 物质的加入不会影响最终成膜的成分。

\section{2 表征手段和离子辐照实验}

采用光学显微镜(9XB-PC)和 ZEISS EVO 18 型 扫描电子显微镜分析样品的表面质量及截面厚度。 采用日本理学 $\mathrm{D} / \mathrm{max} 2500$ 型 $\mathrm{X}$ 射线衍射仪和掠射 角 $\mathrm{X}$ 射线衍射(GIXRD)仪分析样品的晶体结构。采 用 JEM-2100 透射电子显微镜(TEM)进一步表征材 料的微观结构。采用中国科学院近代物理研究所 $320 \mathrm{kV}$ 高电荷态离子综合研究高压平台对 YSZ 薄 膜在室温条件下进行 $500 \mathrm{keV} \mathrm{Xe}$ 离子辐照, 辐照的 剂量分别为 $0.3 、 1.0 、 1.3 、 5.0 、 15.0 、 22.0 \mathrm{dpa}$ 。

\section{2 结果与讨论}

\section{$2.1 \mathrm{ZrO}_{2}$ 薄膜的工艺优化及表征}

研究发现, 工艺参数对 YSZ 纳米晶薄膜的质量 
影响非常大。PVA 作为分散剂加入溶胶可以明显提 高溶胶的粘度, 方便成膜。图 1(a)为未加入 PVA 的 YSZ 薄膜的形貌, 可以看出薄膜表面出现大量的裂 纹, 而添加 PVA 大大提高了成膜质量。研究还发现 随着匀胶转速的提高, 薄膜表面的裂纹逐渐减少, 表面均匀性也有所提高。

薄膜旋涂后的干燥方式也非常重要。研究发现 自然干燥的样品在中心部分存在一些裂纹(图 1(b)), 而经过分级干燥的样品则没有出现裂纹。采用最优 化工艺制备的 YSZ 薄膜的 SEM 形貌如图 1(c)所示, 薄膜表面十分平整, 无明显裂纹。图 1(d)为 YSZ 薄 膜的背散射 SEM(BSD)照片, 可以看出样品的不同 区域几乎没有成分差异。图 2 为样品的截面 SEM 照 片, 薄膜的厚度约为 $60 \mathrm{~nm}$ 。
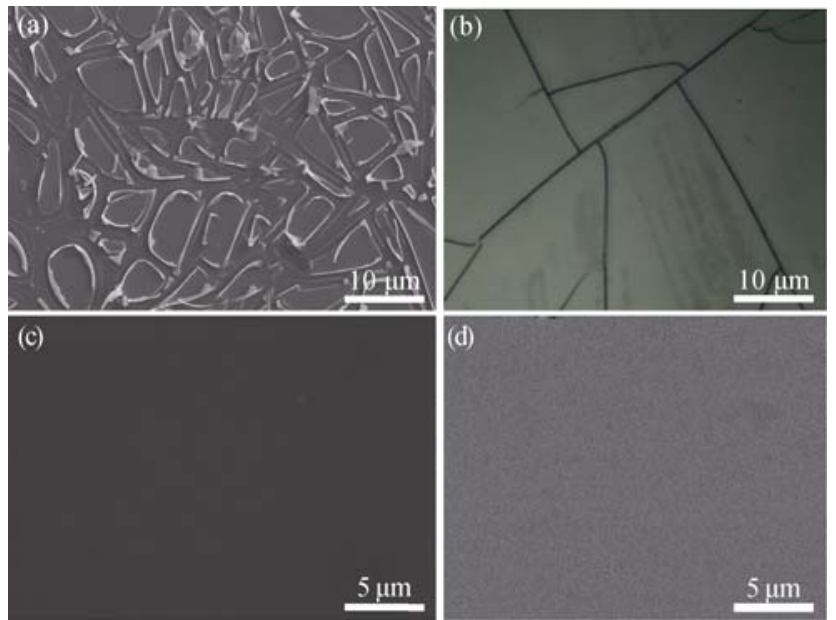

图 1 (a)未加入 PVA 的 YSZ 薄膜的 SEM 形貌和(b)自然干 燥条件下 YSZ 薄膜的光学显微镜照片, 最优化工艺条件下 YSZ 薄膜的 SEM 形貌(c)和 BSD 图片(d)

Fig. 1 (a) SEM image of YSZ film without adding PVA, (b) optical microscope image of YSZ film dried in air, SEM image (c) and BSD image (d) of YSZ film without cracks prepared under optimal conditions

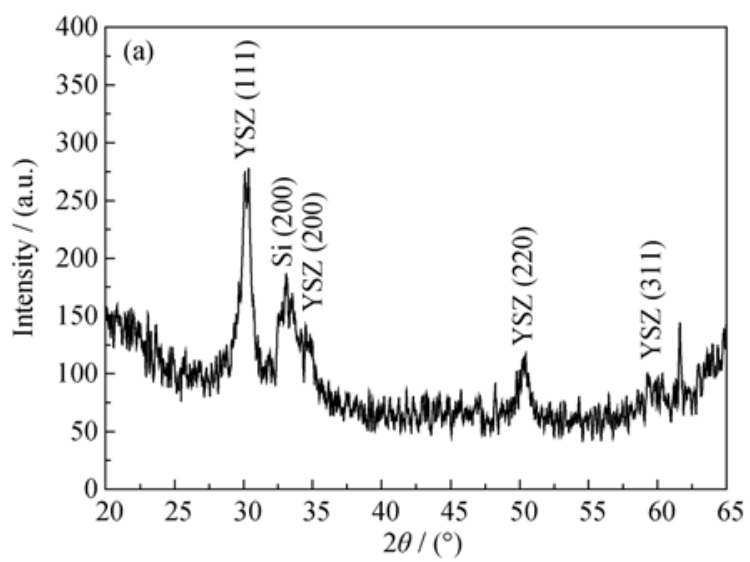

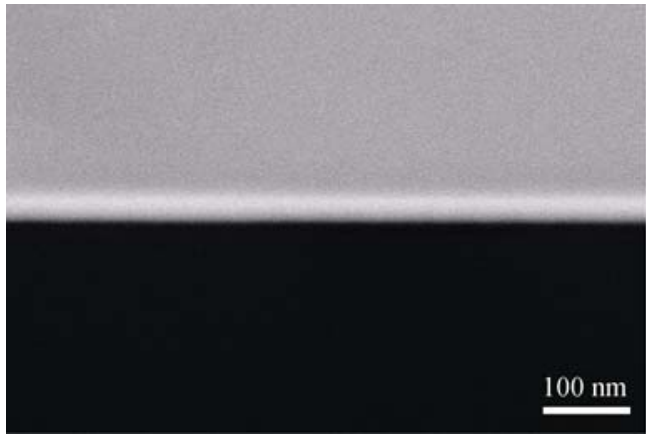

图 2 样品的截面 SEM 照片

Fig. 2 Cross-sectional SEM image of the YSZ film

采用 $\mathrm{X}$ 射线衍射仪分析薄膜样品的相结构, 结 果如图 3(a)所示, 样品的衍射峰分别位于 $2 \theta=30.4^{\circ}$ 、 $52.8^{\circ}$ 和 $59.4^{\circ}$ 处, 与立方相 $\mathrm{ZrO}_{2}$ 的标准 PDF 卡片 (82-1246)的峰位相同，这说明制备的产物为立方 相 $\mathrm{ZrO}_{2}$ 。薄膜中并没有出现非晶化现象以及其他 杂相。图 3(a)中在 $33.16^{\circ}$ 处出现了基底 $\mathrm{Si}$ 的(200) 衍射峰, 这是由于制备的薄膜厚度较薄, $X$ 射线穿 透薄膜与单晶 $\mathrm{Si}$ 基底发生衍射所致。为了排除 $\mathrm{Si}$ 基底对 YSZ 薄膜 XRD 图谱的干扰, 实验还采用 GIXRD 方法分析了相结构, 入射角度为 $0.5^{\circ}$, 结果 如图 3(b)所示。衍射峰全部来自 YSZ 的信号, 所有 衍射峰的位置与立方相 $\mathrm{ZrO}_{2}$ 的标准 PDF 卡片 (82-1246)相同, 可以确定产物为立方相 $\mathrm{ZrO}_{2}$ 。采用 Scherrer 公式来估算晶粒尺寸，由图 3(b) 可知 $2 \theta_{(111)}=30.468^{\circ}, \mathrm{FWHM}_{(111)}=0.885^{\circ}$ ，以(111)晶面 计算得到 YSZ 薄膜中晶粒的大小约为 $9.4 \mathrm{~nm}$, 晶 格常数为 $0.508 \mathrm{~nm}$ 。

图 4(a)为 YSZ 薄膜的剖面 TEM 照片, 可以看出 单晶 $\mathrm{Si}$ 片表面有一层约 2 3 nm 厚的非晶 $\mathrm{SiO}_{2}, \mathrm{YSZ}$ 纳米晶薄膜生长在 $\mathrm{SiO}_{2}$ 层上, 薄膜厚度约为 $60 \mathrm{~nm}$, 纳米晶粒取向明显。 $\mathrm{SiO}_{2}$ 层和 YSZ 纳米晶薄膜层

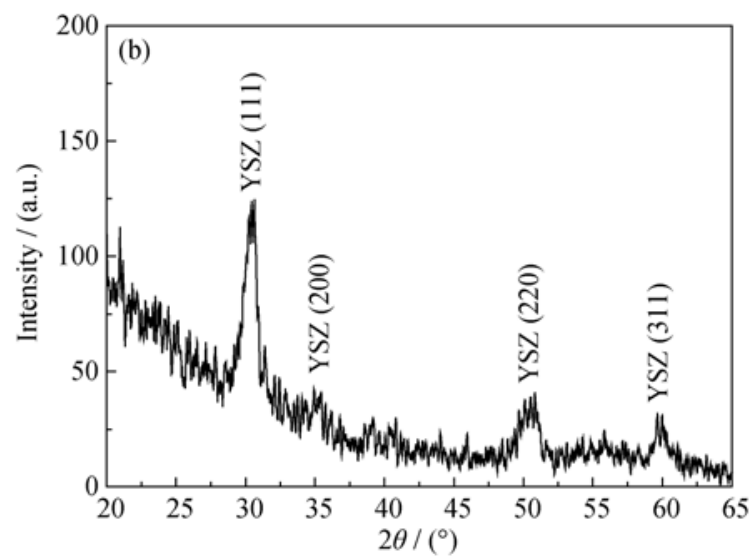

图 3 YSZ 纳米晶薄膜的(a)XRD 和(b)GIXRD 图谱

Fig. 3 (a) XRD and (b) GIXRD patterns of the nanocrystalline YSZ film 
结合较紧密。图 4(b)为图 4(a)的选区电子衍射(SEAD) 图案, 表明产物具有立方相结构。图 4(c)为 YSZ 薄 膜剖面从薄膜到硅片的成分分布曲线, (d)为(c)的局 部放大图, 可以看出 YSZ 纳米晶薄膜与硅祄底有明 显的分界面, 同时也可以看出纳米晶薄膜样品的厚 度约为 $60 \mathrm{~nm}$ 。

\section{$2.2 \mathrm{ZrO}_{2}$ 薄膜的离子辐照效应}

YSZ 薄膜经过不同剂量的 Xe 离子辐照后发生了 比较明显的变化, 结果如图 5 所示。辐照之前, 薄膜 表面非常平整光滑(图 5(a))。经过 $0.3 \mathrm{dpa}$ 剂量辐照后, 表面变化不是很明显, 个别的地方出现裂纹(图 $5(b))$ 。当辐照剂量增加到 $1.0 \mathrm{dpa}$ 时, 薄膜出现明显 的裂纹(图 5(c)), 这可能是由于辐照引起的热峰效应 造成的。YSZ 薄膜与硅祄底的热导率不同, 辐照瞬间 热量很高, 而后又迅速冷却, 导致薄膜出现裂纹。图 6(a)为图 5(c)二次电子像的放大图, 图 6(b)为对应的
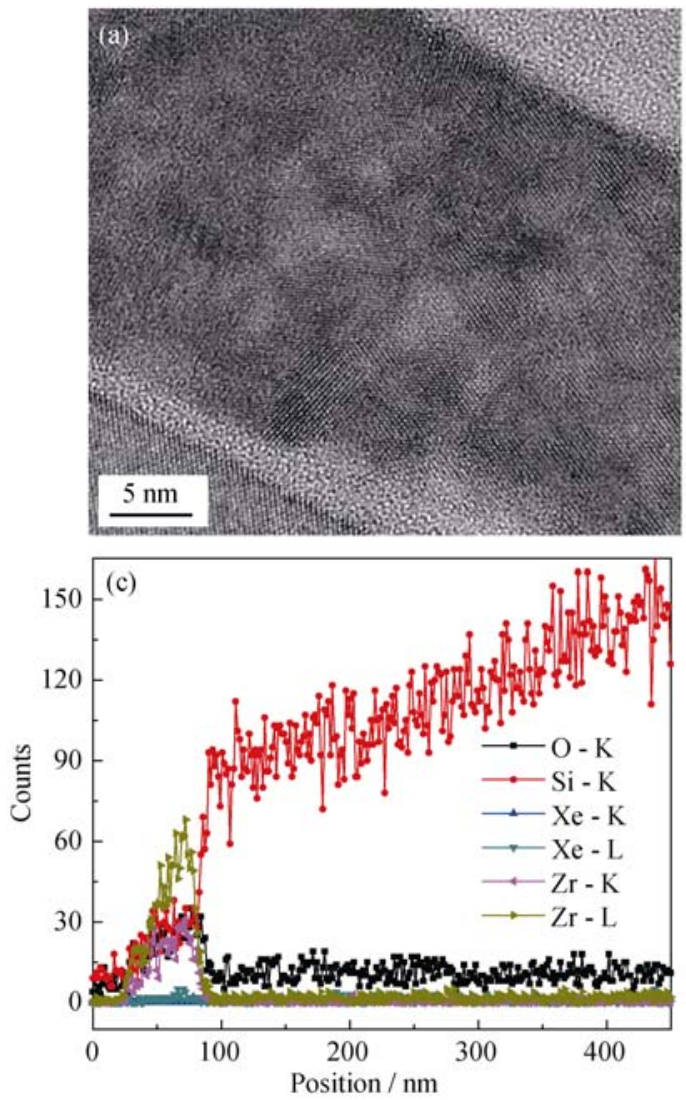

背散射电子图像。沿着裂纹的方向做线扫描成分测 试(如图 6(b)的箭头所示)得到图 6(c)，可以看到图 6(a) 中黑线区域 Si 的信号比较强。另外, 分别在样品的 平整处和突起处测其成分, 发现突起处 $\mathrm{Si}$ 的信号较 强(表 1), 这与在裂纹附近的线扫描成分分析结果相 吻合(图 6(c))。当辐照剂量达到 $1.3 \mathrm{dpa}$ 时, 裂纹的数 量有一定程度的减少，部分裂纹已经开始愈合; 当 辐照计量达到 $5.0 \mathrm{dpa}$ 时, 大部分裂纹消失; 当辐照 剂量为 15.0 和 $22.0 \mathrm{dpa}$ 时, 在 YSZ 薄膜中已经观察 不到裂纹(图 5(d) (f))。这可能是由于辐照剂量较高 时, 在 YSZ 薄膜内产生的热量较多, 辐照发生时间 比较长, 瞬间的热量持续释放, 使得裂纹逐渐愈合。 为了验证这种推测, 实验测试了不同辐照条件下 YSZ 薄膜的晶粒度, 发现薄膜的平均晶粒度随着辐 照剂量的增加而逐渐增大(图 7), 这说明辐照的热峰 效应对薄膜的影响较大。
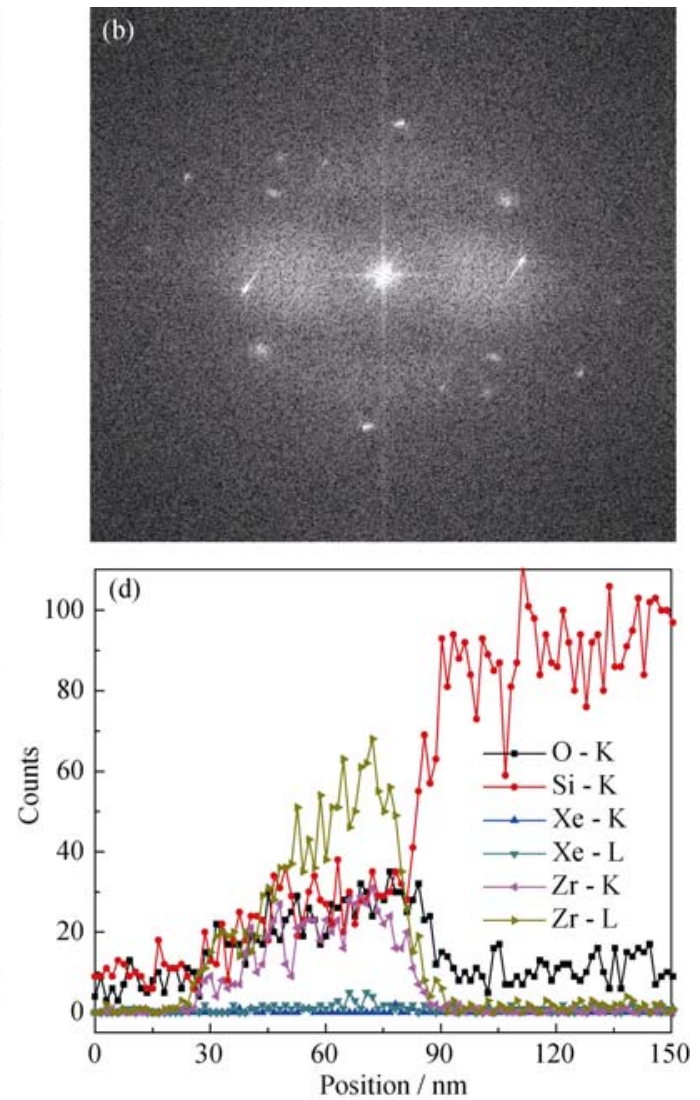

图 4 YSZ 薄膜样品的剖面 TEM 照片(a)及对应区域的 SEAD 图案(b), STEM 模式下从 YSZ 到硅片的成分分布 (c)及其部分放大 $(\mathrm{d})$

Fig. 4 Cross-sectional TEM image (a) and corresponding SAED pattern (b) of YSZ film, STEM-EDS line scan profile from the YSZ film to Si substrate (c) and its enlarged part (d) 

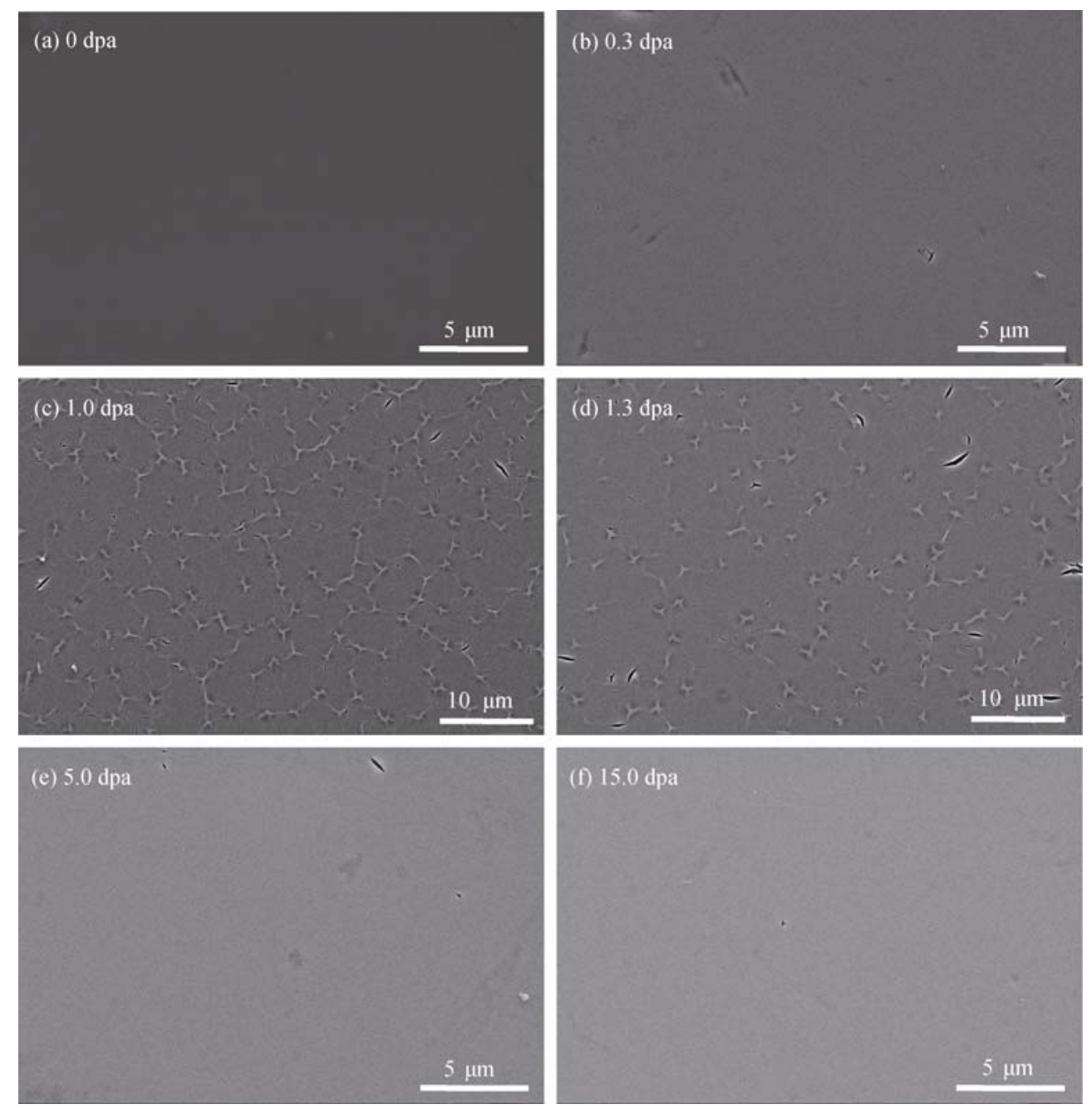

图 5 YSZ 薄膜经不同剂量 Xe 离子辐射后的 SEM 照片

Fig. 5 SEM images of the YSZ films irradiated with different irradiation doses
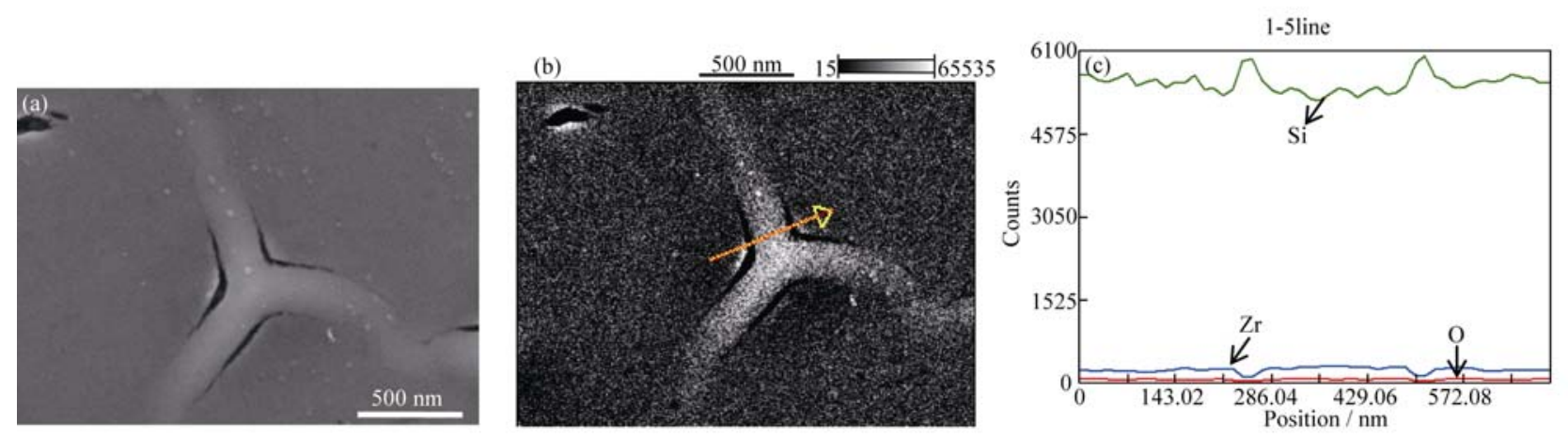

图 $6 \mathrm{YSZ}$ 薄膜在 $1.0 \mathrm{dpa}$ Xe 离子辐照剂量下的高倍 SEM 照片(a)及其对应的 BSD 图片(b)和沿裂纹方向的 EDS 线扫描 分析曲线(c)

Fig. 6 High magnification SEM image of the YSZ films irradiated with 1.0 dpa irradiation dose (a), and corresponding BSD image (b) as well as its EDS line scan profile across the crack (c) 
表 1 样品表面平整处和突起处的元素成分

Table 1 Element compositions at flat and crack position on the surface of sample

\begin{tabular}{ccc}
\hline Element line & Flat site $/$ at\% & Crack site $/$ at\% \\
\hline O K & 17.00 & 14.22 \\
Si K & 79.91 & 83.25 \\
Zr L & 3.10 & 2.53 \\
Total & 100.00 & 100.00 \\
\hline
\end{tabular}

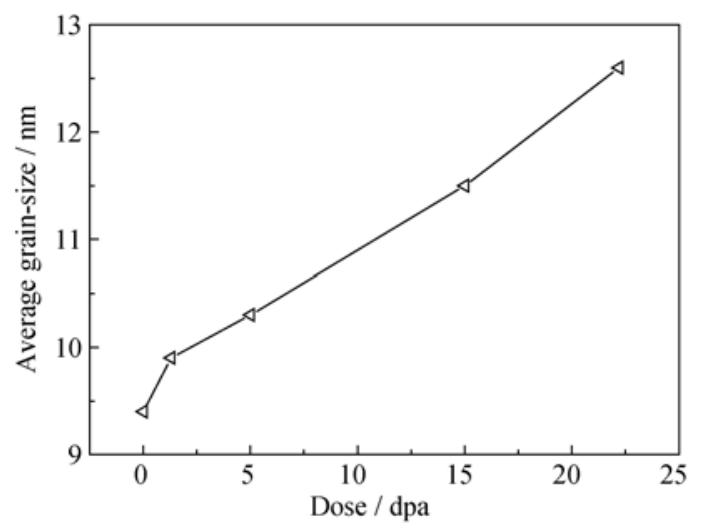

图 7 晶粒尺寸随辐照剂量变化关系

Fig. 7 Plot of average grain-size as a function of irradiation dose

\section{3 结论}

采用溶胶一凝胶结合旋涂法, 通过优化工艺参 数制备了均匀无裂纹的 YSZ 薄膜, 薄膜具有立方相 结构, 厚度约为 $60 \mathrm{~nm}$ 。薄膜由平均尺度约 $9.4 \mathrm{~nm}$ 的纳米晶组成。YSZ 薄膜在室温条件下采用 $\mathrm{Xe}$ 离 子进行辐照, 辐照后 YSZ 薄膜出现微裂纹。当辐照 剂量较高时, 由于热峰效应比较大, 辐照引起的微 裂纹逐渐发生愈合。并且, 随着辐照剂量的增加, YSZ 薄膜纳米晶的平均晶粒尺寸也增大。

\section{参考文献:}

[1] SICKAFUS K E, MATZKE H, HARTMANN T, et al. Radiation damage effects in zirconia. Journal of Nuclear Materials, 1999, 274(1): 66-77.

[2] SASAJIMA N, MATSUI T, HOJOU K, et al. Radiation damage in yttria-stabilized zirconia under Xe ion irradiation. Nuclear Instruments and Methods in Physics Research Section B: Beam Interactions with Materials and Atoms, 1998, 141(1): 487-493.

[3] EDMONDSON P D, WEBER W J, NAMAVAR F, et al. Determination of the displacement energies of $\mathrm{O}, \mathrm{p}[\mathrm{Si}$ and $\mathrm{Zr}$ under electron beam irradiation. Journal of Nuclear Materials, 2012, 422(1): 86-91.

[4] ACKLAND G. Controlling radiation damage. Science, 2010, 327(5973): 1587-1588.

[5] ZHANG YAN-WEN, JIANG WEI-LIN, WANG CHONG-MIN, et al. Grain growth and phase stability of nanocrystalline cubic zirconia un- der ion irradiation. Physical Review B, 2010, 82(18): 184105.

[6] EDMONDSON P D, WEBER W J, NAMAVAR F, et al. Lattice distortions and oxygen vacancies produced in $\mathrm{Au}^{+}$-irradiated nanocrystalline cubic zirconia. Scripta Materialia, 2011, 65(8): 675-678.

[7] YANG TENG-FEI, HUANG XUE-JUN, WANG CHEN-XU, et al. Enhanced structural stability of nanoporous zirconia under irradiation of He. Journal of Nuclear Materials, 2012, 427(1): 225-232.

[8] LU FENG-YUAN, ZHANG JIA-MING, HUANG MENG-BING, et al. Phase transformation of nanosized $\mathrm{ZrO}_{2}$ upon thermal annealing and intense radiation. The Journal of Physical Chemistry C, 2011, 115(15): 7193-7201.

[9] HUANG R T, SHEN Y H, HUANG R H, et al. Characterization of the irradiation-induced phase transition in the monoclinic polymorph of zirconia. Nuclear Instruments and Methods in Physics Research Section B: Beam Interactions with Materials and Atoms, 2014, 332: 293-297.

[10] SHARMA A, VARSHNEY M, SHIN H, et al. Monoclinic to tetragonal phase transition in $\mathrm{ZrO}_{2}$ thin films under swift heavy ion irradiation: structural and electronic structure study. Chemical Physics Letters, 2014, 592: 85-89.

[11] ROSE M, GORZAWSKI G, MIEHE G, et al. Phase stability of nanostructured materials under heavy ion irradiation. Nanostructured Materials, 1995, 6(5): 731-734.

[12] LU FENG-YUAN, WANG JIAN-WEI, LANG M, et al. Amorphization of nanocrystalline monoclinic $\mathrm{ZrO}_{2}$ by swift heavy ion irradiation. Physical Chemistry Chemical Physics, 2012, 14(35): 12295-12300.

[13] MELDRUM A, BOATNER L A, EWING R C. Size effects in the irradiation-induced crystalline-to-amorphous transformation. $\mathrm{Nu}$ clear Instruments and Methods in Physics Research Section B: Beam Interactions with Materials and Atoms, 2003, 207(1): 28-35.

[14] MELDRUM A, BOATNER L A, EWING R C. Nanocrystalline zirconia can be amorphized by ion irradiation. Physical review letters, 2001, 88(2): 025503.

[15] LIAN JIE, ZHANG JIA-MING, NAMAVAR F, et al. Ion beaminduced amorphous-to-tetragonal phase transformation and grain growth of nanocrystalline zirconia. Nanotechnology, 2009, 20(24): 245303.

[16] YANG HUA-MING, OUYANG JING, ZHANG XIAO-LONG, et al. Synthesis and optical properties of yttria-doped $\mathrm{ZrO}_{2}$ nanopowders. Journal of Alloys and Compounds, 2008, 458(1): 474-478.

[17] LIN CUI-KUN, ZHANG CUI-MIAO, LIN JUN. Phase transformation and photoluminescence properties of nanocrystalline $\mathrm{ZrO}_{2}$ powders prepared via the pechini-type Sol-Gel process. The Journal of Physical Chemistry C, 2007, 111(8): 3300-3307.

[18] FÁBREGAS I O, LAMAS D G. Parametric study of the gel-combustion synthesis of nanocrystalline $\mathrm{ZrO}_{2}$-based powders. Powder Technology, 2011, 214(2): 218-228.

[19] MEHNER A, KLÜMPER-WESTKAMP H, HOFFMANN F, et al. Crystallization and residual stress formation of Sol-Gel-derived zirconia films. Thin Solid Films, 1997, 308: 363-368. 